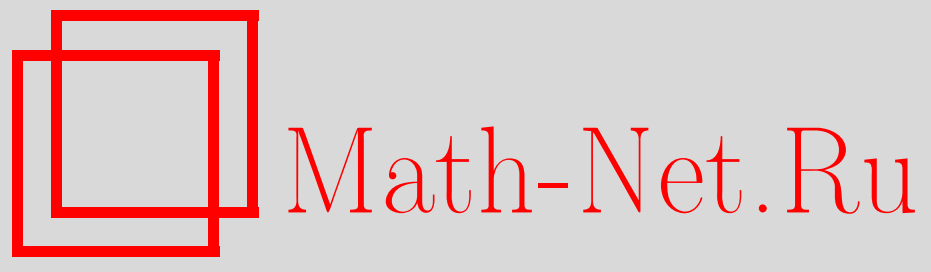

В. А. Юдин, О многочленах, наименее уклоняющихся от нуля, Матем. заметки, 2010, том 87, выпуск 5, 796-800 DOI: https://doi.org/10.4213/mzm8723

Использование Общероссийского математического портала Math-Net.Ru подразумевает, что вы прочитали и согласны с пользовательским соглашением http: //www . mathnet.ru/rus/agreement

Параметры загрузки:

IP: 54.162 .127 .20

26 апреля 2023 г., $12: 46: 34$

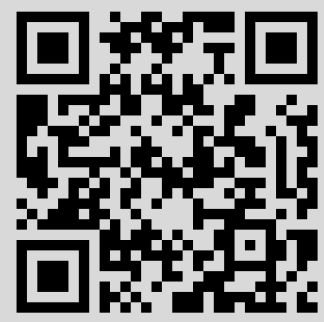




\section{О многочленах, наименее уклоняющихся от нуля}

\section{В. А. Юдин}

Алгебраический многочлен $h(x)=a_{0}+a_{1} x^{1}+\cdots+a_{q} x^{q}$ со старшим коэффициентом $a_{q}$, равным единице, называется наименее уклоняющимся от нуля в пространстве $L_{p}(-1,1)$, если его норма

$$
\|h(x)\|_{p}=\left\{\int_{-1}^{1}|h(x)|^{p} d x\right\}^{1 / p}, \quad 1 \leqslant p \leqslant \infty,
$$

принимает наименьшее значение. При $p=\infty, p=1$ они были построены Чебышевым [1] и в совместной работе [2] Коркиным и Золотарёвым:

$$
T_{q}(x)=2^{1-q} \cos q \alpha, \quad U_{q}(x)=2^{-q} \frac{\sin (q+1) \alpha}{\sin \alpha}, \quad \cos \alpha=x .
$$

Для любого $q \in \mathbb{N}$ доказана их единственность. Они имеют ряд замечательных свойств [1]-[3], нам потребуются следующие три.

1) Все нули $T_{q}(x), U_{q}(x)$ расположены на $[-1,1]$.

2) Коэффициенты при старших степенях $T_{q}(x), U_{q}(x)$ равны единице, причем справедливы разложения

$$
T_{q}(x)=x^{q}-\frac{q}{4} x^{q-2}+\cdots, \quad U_{q}(x)=x^{q}-\frac{q-1}{4} x^{q-2}+\cdots .
$$

Многочлены $T_{q}(x), U_{q}(x)$ с четными $q$ - четные функции, а с нечетными $q$ - нечетные.

3) При любых $q$ из $\mathbb{N}$ справедливы равенства

$$
\int_{-1}^{1} x^{j} \operatorname{sgn} U_{q}(x) d x=0, \quad j=0,1, \ldots, q-1, \quad j=q+1 .
$$

Через $P_{q, q^{\prime}}, q \leqslant q^{\prime}$, обозначим совокупность многочленов степени $q^{\prime}$, у которых коэффициенты при $x^{q}$ равны единице. Пусть

$$
E\left(q, q^{\prime}\right)=\inf _{h \in P_{q, q^{\prime}}}\|h(x)\|,
$$

а $H\left(q, q^{\prime}\right)$ есть все множество многочленов $h(x)$ из $P_{q, q^{\prime}}$, реализующих нижнюю грань в (1). Как и при $q=q^{\prime}$, совокупность $H\left(q, q^{\prime}\right)$ назовем многочленами, наименее уклоняющимися от нуля. Равенство $E(q, q)=E(q, q+1)$ справедливо для всех $p \geqslant 1$. Монотонность $E\left(q, q^{\prime}\right) \leqslant E(q, q)$ очевидна, а противоположное неравенство $E(q, q+1) \geqslant E(q, q)$ вытекает из простых аргументов: четную функцию достаточно приближать четными многочленами, а нечетную - нечетными. Пусть $q=2 k$ четно, тогда для любого $h(x)$ из $P_{q, q+1}$ имеет место $\|h(x)\|=\|h(-x)\|$. Следовательно, по неравенству Минковского

$$
\|h(x)\|=\frac{1}{2}(\|h(x)\|+\|h(-x)\|) \geqslant\left\|\frac{1}{2}(h(x)+h(-x))\right\| .
$$

Многочлен $(1 / 2)(h(x)+h(-x))$ четный. Следовательно, он принадлежит $P_{q, q}$; таким образом, $E(2 k, 2 k+1) \geqslant E(2 k, 2 k)$. Подобным образом равенство $E(q, q+1)=E(q, q)$ устанавливается и для нечетных $q$. При $p=\infty, p=1$ получаем одно и то же значение $E(q, q+1)=2^{1-q}, q \in \mathbb{N}$.

Основной целью работы является описание множеств $H_{q, q+1}$ для $L_{\infty}$ и $L_{1}$. Между $p=\infty$ и $p=1$ наблюдаются следующие отличия:

Работа выполнена при поддержке Российского фонда фундаментальных исследований (грант № 08-01-00598).

(C) В. А. Юдин, 2010 
а) для любых $q \in \mathbb{N}, q \geqslant 2$ и $p=\infty$ многочлен наименьшего уклонения единственен, т.е. $H_{q, q+1}$ состоит всего из одного многочлена $T_{q}(x)$; случай $q=1$ является исключительным, единственность отсутствует;

б) для любых $q \in \mathbb{N}$ и $p=1$ многочлен наилучшего приближения неединственен.

В дальнейшем будем рассматривать только действительный случай, все коэффициенты многочленов действительны. Вначале рассмотрим пространство $L_{\infty}$ и разберем случай $q=1$.

УтвеРЖДЕниЕ 1. Совокупностъю $H_{1,2}$ является множество многочленов

$$
x+\frac{\lambda}{2}\left(1-x^{2}\right), \quad|\lambda| \leqslant 1 .
$$

ДоказАтельство. Так как для любых действительных чисел $a, b$ и $x \in[-1,1]$ справедливы неравенства

$$
\begin{aligned}
\left|a x+\frac{b}{2}\left(1-x^{2}\right)\right| & \leqslant \max \{|a|,|b|\} \cdot\left[|x|+\frac{1-x^{2}}{2}\right] \\
& =\max \{|a|,|b|\} \cdot\left\{1-\frac{1}{2}(1-|x|)^{2}\right\} \leqslant \max \{|a|,|b|\},
\end{aligned}
$$

то, выбирая $a=1$ и $b=\lambda$, получаем (2), т.е. семейство (2) принадлежит $H_{1,2}$. Установим обратное: любой многочлен из $H_{1,2}$ представим в виде (2). Предположим, что с некоторыми $c, d$ выполнено $\max _{|x| \leqslant 1}\left|x+c+d x^{2}\right| \leqslant 1$ или

$$
-1 \leqslant x+c+d x^{2} \leqslant 1, \quad-1 \leqslant x \leqslant 1 .
$$

В левой части неравенства положим $x=-1$, а в правой $-x=1$. Получим $c+d \geqslant 0$ и $c+d \leqslant 0$. Заменяя $d$ на $-c$, приходим к новым неравенствам

$$
c(1+x) \leqslant 1, \quad-1 \leqslant c(1-x), \quad-1 \leqslant x \leqslant 1,
$$

из которых следует, что $|c| \leqslant 1 / 2$. Утверждение доказано.

Для описания $H_{q, q+1}, p=\infty, q \geqslant 2$, предварительно установим следующее.

Теорема 1. Пусть $q \in \mathbb{N}$ и для некоторого тригонометрического полинома

$$
t(\alpha)=\sum_{k=0, k \neq q}^{2 q-1} a_{k} \cos k \alpha
$$

с действительными коэффициентами выполнено неравенство

$$
|\cos q \alpha+t(\alpha)| \leqslant 1, \quad \alpha \in \mathbb{R} .
$$

Тогда $t(\alpha) \equiv 0$.

ДокАзАтЕльство. Полагая $\lambda_{\nu}=a_{\nu}-a_{2 q-\nu}, \mu_{\nu}=a_{\nu}+a_{2 \nu-1}, \nu=1, \ldots, q-1$, преобразуем $t(\alpha)$ и найдем

$$
\begin{aligned}
t(\alpha) & =\frac{1}{2} \sum_{\nu=1}^{q-1} \lambda_{\nu}(\cos \nu \alpha-\cos (2 q-\nu) \alpha)+\mu_{\nu}(\cos \nu \alpha+\cos (2 q-\nu) \alpha) \\
& =\sin q \alpha \sum_{\nu=1}^{q-1} \lambda_{\nu} \sin \nu \alpha+\cos q \alpha \cdot \sum_{\nu=1}^{q-1} \mu_{\nu} \cos \nu \alpha=\sin q \alpha B(\alpha)+\cos q \alpha A(\alpha) .
\end{aligned}
$$


В неравенстве (3) положим $\alpha=\pi j / q, \alpha=[\pi(q-j)] / q, j=0,1, \ldots, q-1$, получим две серии неравенств

$$
\left|1+\sum_{\nu=1}^{q-1} \mu_{\nu} \cos \frac{\nu \pi j}{2}\right| \leqslant 1, \quad\left|1-\sum_{\nu=1}^{q-1} \mu_{\nu} \cos \frac{\nu \pi j}{2}\right| \leqslant 1,
$$

откуда найдем

$$
\sum_{\nu=1}^{q-1} \mu_{\nu} \cos \frac{\nu \pi j}{q}=0, \quad j=0,1, \ldots, q-1 .
$$

Следовательно, у тригонометрического полинома $A(\alpha)$ порядка $q-1$ на $T(T=\mathbb{R} / 2 \pi \mathbb{Z})$ имеется по крайней мере $2 q-1$ нулей, что возможно лишь при $A(\alpha) \equiv 0$. Из представления (4) находим

$$
t(\alpha)=\sin q \alpha \sum_{\nu=1}^{q-1} \lambda_{\nu} \sin \nu \alpha .
$$

Поскольку в точках $j \pi / q, j=0,1, \ldots, 2 q-1, \cos q \alpha$ поочередно принимает свои наибольшее и наименьшее значения \pm 1 , то из (3) получим, что в этих точках производная $t^{\prime}(\alpha)$ обязана обращаться в нуль. Так как

$$
t^{\prime}(\alpha)=q \cos q \alpha \sum_{\nu=1}^{q-1} \lambda_{\nu} \sin \nu \alpha+\sin q \alpha \cdot \sum_{\nu=1}^{q-1} \nu \lambda_{\nu} \cos \nu \alpha,
$$

то

$$
\sum_{\nu=1}^{q-1} \lambda_{\nu} \sin \frac{\nu \pi}{q} j=0, \quad j=0,1, \ldots, 2 q-1 .
$$

Полином $B(\alpha)$ имеет лишний нуль. Значит, $B(\alpha) \equiv 0$ и, следовательно, $t(\alpha) \equiv 0$. Теорема доказана.

ЗАмЕчАниЕ. Если тригонометрический полином $t(\alpha)$ из (3) имеет порядок, больший чем $2 q-1$, то $t(\alpha)$ не обязан обращаться в нуль. Положим в $(2) x=\cos q \alpha$, получим

$$
\left|\cos q \alpha+\frac{\lambda}{2} \sin ^{2} q \alpha\right|=\left|\cos q \alpha+\frac{\lambda}{4}-\frac{\lambda}{4} \cos 2 q \alpha\right| \leqslant 1, \quad \alpha \in T,
$$

для любого $\lambda,|\lambda| \leqslant 1$. Отметим, что в качестве $\lambda$ можно взять произвольный тригонометрический полином порядка $q-1$, у которого норма в $C[T]$ не превосходит единицы.

СлЕДСтвиЕ 1. Для $q \geqslant 2$ в пространстве $C[-1,1]$ алгебраический многочлен наименъшего уклонения из $P_{q, q+1}$ единственен.

Пусть $x^{q}+h(x)$ принадлежит $H_{q, q+1}$, положим $x=\cos \alpha$. Из свойства 2 находим,

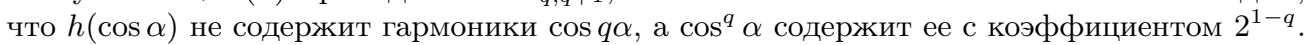
Поэтому с некоторыми коэффициентами $\left\{a_{\nu}\right\}_{0}^{q+1}, \nu \neq q$, справедливо неравенство

$$
\left|2^{1-q} \cos q \alpha+\sum_{\nu=0, \nu \neq q}^{q+1} a_{\nu} \cos \nu \alpha\right| \leqslant 2^{1-q} .
$$

Так как при $q \geqslant 2, q+1 \leqslant 2 q-1$, то из (3) находим, что $x^{q}+h(x)=T_{q}(x)$.

Для описания $H_{q, q+1}$ в пространстве $L(-1,1)$ потребуется

УТВЕРЖДЕНИЕ 2. Пусть

1) $f(x), g(x)$ - непрерывные функиии с конечным числом нулей на $[-1,1]$; 
2) имеет место равенство

$$
\int_{-1}^{1} f(x) \operatorname{sgn} g(x) d x=\int_{-1}^{1}|f(x)| d x .
$$

Тогда функиии $f(x)$ и $g(x)$ имеют одинаковые знаки на $[-1,1]$.

Поскольку

$$
\int_{-1}^{1} f(x) \operatorname{sgn} g(x) d x=\int_{-1}^{1}|f(x)| \operatorname{sgn} f(x) \operatorname{sgn} g(x) d x=\int_{-1}^{1}|f(x)| \operatorname{sgn}[f(x) g(x)] d x,
$$

то в силу второго условия $(5)$ имеем $f(x) g(x)>0$ на $(-1,1)$ за исключением конечного множества точек.

Имеет место

Теорема 2. Любой многочлен из $H_{q, q+1}$ в пространстве $L(-1,1)$ имеет вид

$$
(1+\lambda x) U_{q}(x), \quad|\lambda| \leqslant 1 .
$$

ДокАЗАтЕльСтво. В силу второго свойства $U_{q}(x)$ коэффициент при $x^{q}$ у произведения $x U_{q}(x)$ равен нулю, а у $U_{q}(x)$ он равен единице. Значит, многочлен (6) входит в $P_{q, q+1}$. Вычислим его норму. Так как $\left|U_{q}(x)\right|$ есть четная функция, а $1+\lambda x \geqslant 0$ на $[-1,1]$, то

$$
\int_{-1}^{1}\left|(1+\lambda x) U_{q}(x)\right| d x=\int_{-1}^{1}(1+\lambda x)\left|U_{q}(x)\right| d x=\int_{-1}^{1}\left|U_{q}(x)\right| d x=2^{1-q} .
$$

Покажем, что любой многочлен из $H_{q, q+1}$ представим в виде (6). Допустим, что $U_{q}(x)+h(x) \in H_{q, q+1}$ и

$$
\left\|U_{q}(x)+h(x)\right\|_{1}=\int_{-1}^{1}\left|U_{q}(x)+h(x)\right| d x=2^{1-q} .
$$

Из свойства 3 имеем

$$
I=\int_{-1}^{1}\left[U_{q}(x)+h(x)\right] \operatorname{sgn} U_{q}(x) d x=\int_{-1}^{1} U_{q}(x) \operatorname{sgn} U_{q}(x) d x=2^{1-q} .
$$

В (5) положим $f(x)=U_{q}(x)+h(x), g(x)=U_{q}(x)$. Получим, что за исключением конечного множества точек $U_{q}(x)+h(x)$ и $U_{q}(x)$ имеют одинаковые знаки на $[-1,1]$. Следовательно, множество нулей $U_{q}(x)+h(x)$ содержит все нули $U_{q}(x)$. Используя первое свойство $U_{q}(x)$, находим представление

$$
U_{q}(x)+h(x)=U_{q}(x)(a x+b),
$$

где $a x+b \geqslant 0$ на $[-1,1]$. Сравнивая коэффициенты при $x^{q}$ в левой и правой частях равенства, получим $b=1$. Поскольку неравенство $a x+1 \geqslant 0$ на $[-1,1]$ возможно лишь при $|a| \leqslant 1$, то

$$
U_{q}(x)+h(x)=U_{q}(x)(1+\lambda x), \quad|\lambda| \leqslant 1 .
$$

Теорема доказана.

ЗАмечАниЕ. Имеется иной способ доказательства теоремы 2. Отправляясь от точного решения [4], [5] задачи Золотарёва в пространстве $L(-1,1)$

$$
\inf _{a_{j}}\left\|x^{q+1}+\alpha x^{q}+a_{q-1} x^{q-1}+\cdots+a_{a}\right\|_{1}= \begin{cases}\left\|U_{q+1}+\alpha U_{q}+\frac{\alpha^{2}}{4} U_{q-1}\right\|_{1}, & |\alpha| \leqslant 1, \\ \left\|U_{q+1}+\alpha U_{q}+\frac{1}{4} U_{q-1}\right\|_{1}, & |\alpha| \geqslant 1,\end{cases}
$$

В. И. Иванов дал новый вывод представления (6). 


\section{СПИСОК ЦИТИРОВАННОЙ ЛИТЕРАТУРЫ}

[1] П.Л. Чебышев, Полное собрание сочинений, т. 2, Изд-во АН СССР, М., 1947. [2] Е.И. Золотарев, А.И. Коркин: Е.И. Золотарев, Полное собрание сочинений, т. 1, Изд-во АН СССР, Л., 1931, 138-153. [3] Н. И. Ахиезер, Лекчии по теории аппроксимачии, Наука, М., 1965. [4] J. Geronimus, Ann. of Math. (2), 37:2 (1936), 483-517. [5] Э. М. Галеев, Матем. заметки, 17:1 (1975), 13-20.

\section{В. А. Юдин}

Поступило

Московский энергетический институт

26.12.2009 (технический университет)

E-mail: vlayudin@mtu-net.ru, yudin@vayudin.mccme.ru 